\title{
Suivi temporel de mouvements gravitaires: apport des vibrations sismiques ambiantes
}

\author{
Pierre Bottelin $^{1, \star}$, Guénolé Mainsant ${ }^{2}$, Denis Jongmans ${ }^{1}$, Laurent Baillet ${ }^{1}$, Eric Larose ${ }^{1}$, \\ Guillaume Chambon ${ }^{3}$ et Didier Hantz ${ }^{1}$ \\ ${ }^{1}$ Université Grenoble Alpes, CNRS, ISTerre, 38000 Grenoble, France \\ ${ }^{2}$ Géosciences Montpellier, université Montpellier II, CNRS, 34000 Montpellier, France \\ 3 IRSTEA, UR ETGR, 38000 Grenoble, France
}

\begin{abstract}
Résumé - Nous avons enregistré les vibrations sismiques ambiantes durant plusieurs années sur quatre sites naturels d'éboulements rocheux et un glissement de terrain argileux. Quels que soient le volume, la géométrie, le mécanisme de rupture, le matériau ou le type de mouvement, les mesures sismiques ont permis d'obtenir des informations globales sur l'état mécanique de l'instabilité. Pour les éboulements rocheux potentiels, la fréquence de résonance fondamentale du compartiment instable $f_{0}$ a systématiquement été déterminée. Ce paramètre mécanique global dépend de (1) la géométrie, (2) de la densité, (3) de la rigidité interne et (4) de l'accroche du compartiment rocheux au massif stable. En supposant (1), (2) et (3) constants, une diminution irréversible de $f_{0}$ reflète la déstabilisation progressive du compartiment instable avant la rupture. La mesure en continu de $f_{0}$ représente alors un nouveau précurseur aux éboulements. Cependant, des fluctuations réversibles parfois importantes affectent $f_{0}$. La dynamique de ces variations est contrôlée par la température, la déstructuration et dépend de la période (de journalière à saisonnière). Les simulations numériques entreprises montrent que la contraction-dilatation du matériau et la dépendance de son module élastique à la température permettent d'expliquer ces fluctuations de $f_{0}$. Le glissement de terrain argileux étudié connaît une évolution lente entrecoupée d'épisodes de coulées catastrophiques. Le corps argileux du glissement présente une transition brutale d'un état « solide » à un état « fluide » lorsqu'un seuil de contrainte cisaillante est dépassé. Les études rhéologiques de laboratoire ont montré que la vitesse des ondes sismiques de cisaillement $\left(V_{S}\right)$ diminue notablement avant la transition solide-fluide. Sur le site d'étude, une diminution de $7 \%$ de $V_{S}$ a été observée 20 jours avant le déclenchement d'une coulée argileuse. La déstructuration de l'argile a pu être localisée dans une couche d'environ $2 \mathrm{~m}$ d'épaisseur située la base du glissement.
\end{abstract}

Mots clés : vibrations ambiantes / mouvements de terrain / éboulements / suivi temporel

Abstract - Landslide monitoring: recent learnings from ambient vibrations. Four potential rockfalls and one clayey landslide were monitored using ambient vibrations over many years. Whatever the volume, geometry, rupture mechanism, material or movement type, passive seismic measurements yielded pertinent global information about the mechanical condition of the gravitational instability. For each potential rockfall, we successfully determined the fundamental resonance frequency of the unstable rock compartment (denoted $f_{0}$ ). This global mechanical parameter depends on the unstable compartment's (1) geometry, (2) density, (3) internal rigidity and (4) its bounding stiffness with the nearby stable massif. Assuming (1), (2) and (3) are constant, an irreversible drop in $f_{0}$ reflects the progressive destabilization of the unstable compartment before the rupture. Continuous monitoring of $f_{0}$ hence provides a new precursor to rockfalls. Reversible fluctuations of non-negligible amplitude yet affect $f_{0}$ time-series. These variations originate from thermal effects and depend on the air temperature, the rock fracturing and the period of study (daily to yearly periods). Numerical simulations showed that (1) rock contraction/dilation and (2) rock elastic modulus variation with temperature can explain these reversible $f_{0}$ fluctuations. The studied landslide shows a complex temporal evolution, with a relatively slow, steady rate of movements interrupted by dramatic earth-flow episodes. The landslide body is made of finely crushed clayey shale, which shows a sudden transition between a "solid" state and a "fluid" state when increasing the shear stress. Laboratory

\footnotetext{
$\bar{\star}$ Auteur de correspondance : pierre.bottelin.geo@gmail.com
} 
rheological experiments showed that the seismic shear wave velocity $\left(V_{S}\right)$ clearly drops prior to the fluidification. For the monitored landslide, a consistent $7 \%$ drop in $V_{S}$ was observed about 20 days before a catastrophic earth-flow. It was interpreted as a clay fluidification processes affecting a $2 \mathrm{~m}$ thick layer at the base of the landslide body.

Keywords: ambient vibrations / landslides / rockfalls / monitoring

\section{Introduction}

\section{1 Éboulements rocheux}

Les éboulements rocheux intermédiaires $\left(10^{3}\right.$ à $\left.10^{6} \mathrm{~m}^{3}\right)$ sont caractérisés par des fréquences d'occurrence moyennes, une répartition spatiale discrète et un pouvoir destructeur élevé (CFGI, 2000; Agliardi et Crosta 2003 ; Frayssines 2005). Ces évènements sont particulièrement problématiques lorsqu'ils affectent les infrastructures routières, entraînant pertes de vies humaines, dégâts et interruptions de circulation. En France métropolitaine, on peut citer les éboulements récurrents des Gorges de l'Arly (73, Savoie) ou des Gorges de la Bourne (38, Isère) avec deux évènements meurtriers en 2004 et 2007 pour ces dernières. Les infrastructures ferroviaires peuvent également être durement touchées comme lors de l'éboulement de la Clapisse (38, Isère) en 2010: avec un volume éboulé de seulement $3000 \mathrm{~m}^{3}$, l'exploitation du chemin de fer de la Mure n'a toujours pas repris en 2016.

Les éboulements se déclenchent lors d'une augmentation des forces déstabilisatrices ou d'une diminution des actions favorables sur le compartiment rocheux instable. La dynamique de cette déstabilisation progressive dépend de nombreux facteurs (thermique, action de l'eau, cycles geldégel, vibrations, Iverson, 2000; Matsuoka, 2001 ; Cornforth, 2005; Gunzburger et al., 2005; Frayssines et Hantz 2006; Mufundirwa et al., 2011; Hovius et Meunier, 2012) et reste difficile à prédire.

\subsection{Glissements de terrain argileux}

Les glissements de terrain argileux sont souvent caractérisés par des accélérations brutales, aboutissant potentiellement à des coulées argileuses. Ces coulées sont liées à des diminutions rapides des propriétés mécaniques des argiles, généralement causées par des précipitations intenses. En France métropolitaine, de nombreuses régions sont sujettes à ce type de glissements argileux soudains. On peut citer l'évènement de la Salle-en-Beaumont (38, Isère) qui a coûté la vie à 4 personnes en janvier 1994. À l'étranger, ont peut citer les glissements de Guinsaugon (février 2006, Philippines) et Oso (mars 2014, États-Unis) qui ont causé respectivement environ 2000 et 50 décès.

La diminution soudaine des caractéristiques mécaniques des argiles est due à des phénomènes de transition d'un état solide à un état fluide, aussi appelé bifurcation de viscosité (Coussot et al., 2002). Cette transition n'est pas liée à la limite de liquidité géotechnique, définie comme la teneur en eau limite entre l'état plastique et l'état liquide (Casagrande 1932; Cornforth 2005). En effet, les argiles saturées connaissent deux comportements distincts à l'intérieur du domaine de liquidité géotechnique : un état solide (dit aussi «coincé ») où la matière ne s'écoule pas et un état fluide (dit «décoincé») lorsque la matière se déforme et s'écoule. Les paramètres principaux régissant cette transition sont l'état de contraintes appliqué et la teneur en eau, mais d'autres paramètres peuvent influencer le comportement du milieu argileux tels que la température et la fraction volumique des particules (Trappe et al., 2001). Les lois viscoplastiques telle que celle d'Herschel-Bulkley modélisent bien la déstructuration des argiles, pour lesquelles la transition est déterminée par une contrainte cisaillante critique $\tau_{\mathrm{c}}$ (Huang et Garcia, 1998). Au niveau du seuil, les études sur des argiles synthétiques telle que la bentonite ou des argiles naturelles ont montré une bifurcation de viscosité (Coussot et al., 2002; Khaldoun et al., 2009). En deçà de la contrainte seuil, la viscosité décroît avec le temps et l'écoulement initial éventuel s'arrête. En revanche si $\tau_{\mathrm{c}}$ est atteinte, le matériau connaît une chute brutale - mais non instantanée - de sa viscosité qui se stabilise à une valeur fixe traduisant un écoulement soudain. Ce comportement thixotropique peut expliquer les départs rapides et catastrophiques des glissements argileux.

Des études récentes de terrain ont montré que la vitesse des ondes de cisaillement $V_{S}$ est sensible à la rigidité du milieu et sa diminution est susceptible de refléter l'état de déstructuration des argiles (Jongmans et al., 2009; Renalier et al., 2010). Ces études ont été corroborées par les études rhéomètriques que nous avons entreprises sur des échantillons d'argile (Mainsant et al., 2012a).

\subsection{Techniques de surveillance}

Pour les éboulements rocheux intermédiaires et les glissements de terrain argileux, les mesures de protection sont généralement délicates à mettre en oeuvre. D'une part, les volumes élevés mis en jeu rendent les travaux de protection coûteux et peu efficaces, tandis que les fréquences d'occurrence relativement importantes rendent impossible l'évacuation des biens et des personnes dans toutes les zones concernées. De plus, les prévisions suggèrent que les conséquences des instabilités gravitaires pourraient s'aggraver dans les décennies à venir, en raison du développement de l'urbanisation dans les régions montagneuses et des tendances climatiques attendues (Schuster, 1996; Petley, 2013). La solution optimale d'un point de vue budgétaire et de hiérarchisation des priorités semble donc la mise en place de solutions de surveillance des instabilités.

Classiquement, la surveillance des terrains instables repose sur deux méthodologies principales. La première consiste à mesurer en continu les facteurs de déstabilisation potentiels agissant sur les terrains, principalement les précipitations et la température. La seconde consiste à relever les indices d'activité du mouvement de terrain: occurrence de petits évènements précurseurs, déplacement du sol, ouverture des 
fractures (Saito, 1980 ; Fukuzono, 1985 ; Voight, 1989 ; Azimi et Desvarreux, 1996; Rosser et al., 2007). Ces indices peuvent être obtenus via une grande variété de techniques et d'instruments : extensomètres, inclinomètres, levés topographiques et l'acquisition de modèles de terrain. Cette dernière technique a été facilitée par les développements technologiques récents en photogrammétrie, LiDAR (Light Detection And Ranging) ou InSAR (Interferometric Synthetic Aperture Radar) opérés depuis le sol ou aéroportés (Abellán et al., 2009; Oppikofer et al., 2009; Abellán et al., 2010; Bozzano et al., 2010). L'évolution du mouvement de terrain est alors extrapolée à partir des ces mesures d'activité et/ou des facteurs de déstabilisation; via divers modèles physiques ou non physiques (Crozier, 1999; Zvelebill et Moser 2001; Wilkinson et al., 2002; Crosta et Agliardi 2003; Sornette et al., 2004; Suwa et al., 2010). Ces techniques restent cependant limitées car les mesures réalisées sont ponctuelles dans le temps (périodicité des relevés) ou dans l'espace (répartition des points de mesure et des forages). Les mesures les plus courantes et les moins onéreuses restent généralement cantonnées à la surface du sol. Ces limitations ont encouragé la recherche de techniques de surveillance complémentaires afin de disposer de précurseurs additionnels.

Les mesures géophysiques ont été de plus en plus utilisées pour étudier et suivre l'évolution de pentes instables. Des études antérieures ont montré que la géophysique sismique pouvait permettre de détecter et caractériser la déformation du matériau rocheux (Amitrano et al., 2005; Brückl et Mertl, 2006 ; Spillmann et al., 2007 ; Senfaute et al., 2009; Amitrano et al., 2010; Helmstetter et Garambois 2010; Lévy et al., 2011; Occhiena et al., 2012), détecter et localiser de petits éboulements (Vilajosana et al., 2008; Lacroix et Helmstetter, 2011 ) et comprendre la réponse sismique de pentes instables (Burjánek et al., 2010 ; Gaffet et al., 2010 ; Moore et al., 2011 ; Burjánek et al., 2012).

Deux types de méthodes sismiques coexistent pour enregistrer des signaux sismiques se propageant dans un milieu. Le premier groupe recourt à des sources sismiques actives anthropiques (marteau, explosifs, etc.) ou naturelles (séismes). Le second groupe, largement développé ces dix dernières années, consiste à utiliser les vibrations ambiantes aussi appelées bruit de fond sismique. Ces vibrations sont des mouvements de faible ampleur, pseudo-stationnaires, principalement composées d'ondes de surface et présents sur toute la surface du globe (Gutenberg, 1958; Withers et al., 1996; Mucciarelli et al., 2005 ; Bonnefoy-Claudet et al., 2006). Le traitement adapté des vibrations ambiantes permet de s'affranchir de sources sismiques actives. Le poids et le coût d'instrumentation diminuent ainsi sensiblement, tandis que des observations sismologiques en continu et à toutes les échelles deviennent accessibles. Nos travaux de surveillance de mouvements gravitaires, basés sur les vibrations ambiantes, ont été analysées suivant deux techniques: la fréquence de résonance (pour les sites d'éboulements potentiels) et le calcul des corrélations (pour le glissement de terrain argileux).

\subsection{Fréquence de résonance}

La première mesure de fréquences de résonance sur une écaille rocheuse instable a été menée par (Lévy et al., 2010).
Le compartiment instable de $21000 \mathrm{~m}^{3}$ a été instrumenté avec des sismomètres et des extensomètres durant 4 mois. Les enregistrements de vibrations ambiantes ont montré des pics spectraux bien marqués sur le compartiment instable, contrairement au massif stable adjacent. Des modélisations numériques ont montré que ces pics correspondent aux fréquences de résonance de la masse instable. La fréquence de résonance la plus basse et la plus énergétique est appelée fréquence de résonance fondamentale et notée $f_{0}$ (Fig. 1a). Un modèle simple permet d'expliquer ces observations, dans lequel $f_{0}$ dépend de la masse $m$ du compartiment instable, de sa rigidité interne $K_{i}$ et de la rigidité du contact $K_{c}$ (Fig. 1b). $f_{0}$ a été suivie au cours du temps pour détecter d'éventuels changements des propriétés mécaniques du compartiment instable.

Une chute irréversible de fréquence de résonance d'environ $30 \%$ a été observée quelques semaines avant l'éboulement $(\Delta \mathrm{F}$, Fig. 1a) simultanément à l'ouverture de la fracture arrière. Ces observations sont la conséquence probable de la rupture des ponts rocheux qui relient le compartiment instable au massif (Fig. 1b). Cette hypothèse est appuyée par les cassures fraîches observées sur la cicatrice de l'éboulement et par des modélisations numériques dynamiques (Lévy et al., 2010). Cette étude a démontré la possibilité de mesurer des fréquences de résonance sur un objet naturel avec du matériel sismologique. La fréquence fondamentale $f_{0}$ est un paramètre que l'on peut suivre au cours du temps, qui dépend de l'endommagement se produisant sur la future surface de rupture.

\subsection{Corrélation de vibrations ambiantes}

Cette technique sismique nécessite une distribution équipartie des vibrations ambiantes, c'est-à-dire une répartition homogène et aléatoire des vibrations sismiques dans l'espace. Dans ce cas, la corrélation des enregistrements sismiques de deux capteurs disposés à une certaine distance l'un de l'autre permet de reconstruire la propagation des ondes sismiques dans le milieu. Comme les vibrations ambiantes sont principalement composées d'ondes de surface, la corrélation de signaux de capteurs verticaux permet la reconstruction des ondes de Rayleigh. Celles-ci peuvent ensuite être étudiées avec les méthodes d'inversion classiques. Cette technique de corrélation peut être généralisée à un grand nombre de capteurs et utilisée à toutes les échelles. Pour des applications de surveillance, on s'intéresse généralement au suivi des variations relatives de vitesse d'ondes de Rayleigh au cours du temps. Dans ce cas, il n'est pas nécessaire de reconstruire parfaitement l'onde de surface: les vibrations ambiantes peuvent être réparties de manière hétérogène, pour autant qu'elles soient stables dans le temps. Comme la vitesse de ces ondes $V_{R}$ est reliée à la vitesse des ondes de cisaillement par le coefficient de Poisson du matériau (Viktorov, 1970), il est possible de mesurer des variations rigidité du milieu avec simplement deux capteurs.

Cette technique s'est récemment largement développée pour la surveillance de volcans actifs (Brenguier et al., 2008b; Duputel et al., 2009), de zones de failles (Brenguier et al., 2008a), le suivi de sites géothermiques (Obermann et al., 2015) ou de glissements de terrain (Renalier et al., 2010). 


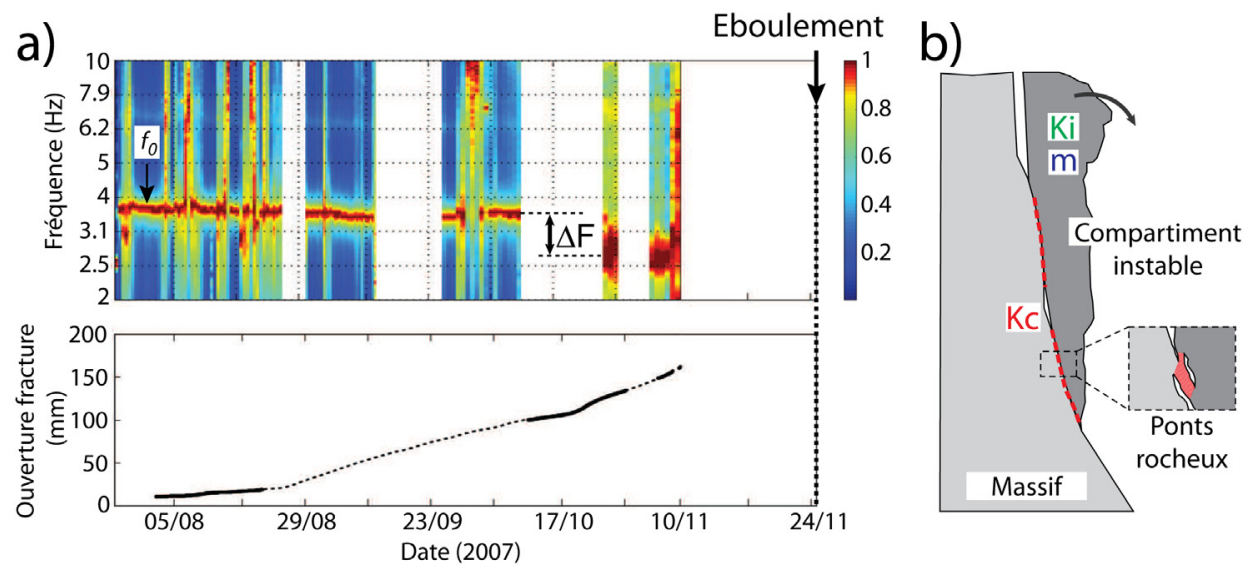

Fig. 1. a : (Haut) Spectres normalisés en fonction du temps pour le sismomètre vertical sur le compartiment instable de Chamousset. Période de juillet à novembre 2007. La fréquence de résonance fondamentale $f_{0}$ apparaît en rouge. La chute de fréquence de résonance fondamentale $\left(f_{0}\right)$ est notée $\Delta F$. (Bas) Ouverture de la fracture arrière sur la même période; b : schéma d'un compartiment rocheux instable. $f_{0}$ est fonction de la géométrie du compartiment, de sa masse $m$, de sa rigidité interne $K_{i}$ et de la rigidité du contact avec le massif $K_{c}$. Adapté de Lévy et al. (2010). Fig. 1. a: (Top) Normalized spectra of vertical seismic records. Chamousset unstable column case study, from July to November 2007. The fundamental resonance frequency $f_{0}$ is shown by the red maxima. The $f_{0}$ drop is labeled $\Delta F$. (Bottom) Rear fracture opening over the same period; $b$ : scheme of an unstable rock compartment (dark grey) decoupling from the stable rock mass (light grey). $f_{0}$ is controlled by the unstable mass $(m)$, its geometry, its internal rigidity $\left(K_{i}\right)$ and the bound stiffness with nearby rock mass $\left(K_{c}\right)$.
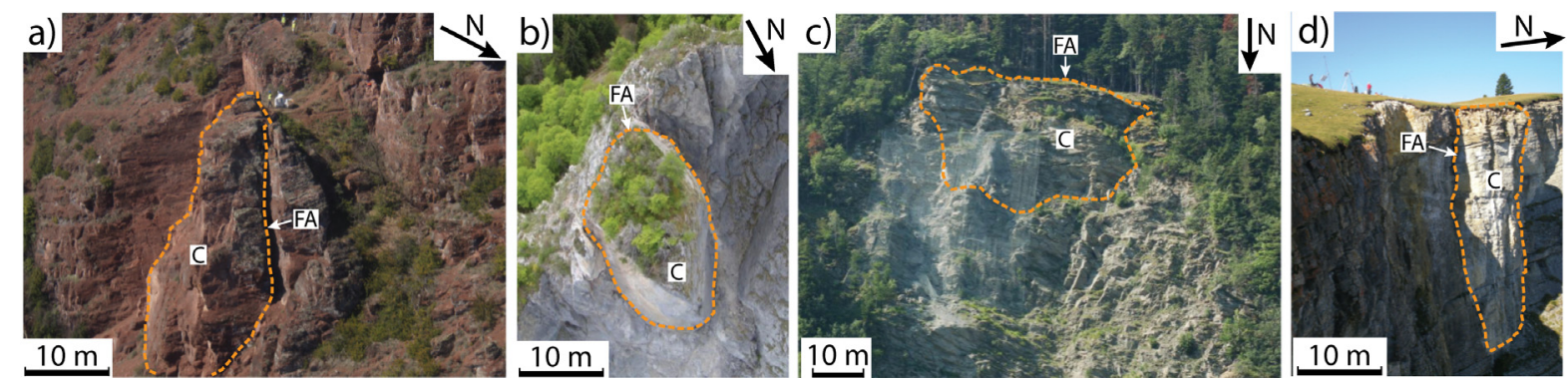

Fig. 2. Photographies des sites d'éboulement potentiels : a : Rubi ; b : La Suche; c : La Praz; d : Les Arches. Les compartiments instables ( $C$ ) sont entourés de pointillés orange. Une flèche montre la principale fracture $(F A)$ les séparant du massif stable.

Fig. 2. Pictures of the four potential rockfall sites: $a$ : Rubi; $b$ : La Suche; $c$ : La Praz; $d$ : Les Arches. The unstable rock compartments (C) are delineated with dashed orange lines. The FA arrow points out the main rear fracture between stable and unstable rock masses.

\subsection{Organisation du manuscrit}

Dans cet article, nous présentons les principales observations découlant du suivi de 5 sites naturels par enregistrement des vibrations ambiantes. Quatre éboulements potentiels de volume intermédiaire et un glissement de terrain argileux ont été instrumentés. Nous détaillons les modélisations numériques ou analogiques permettant d'interpréter ces mesures pour mieux comprendre les mécanismes impliqués dans la déstabilisation des terrains.

\section{2 Éboulements rocheux}

\subsection{Mesures de fréquence de résonance}

Quatre sites d'éboulements potentiels ont été instrumentés à travers les Alpes françaises, italiennes et suisses dans le cadre de nos travaux. Ces sites sont présentés sur la Figure 2. Ils représentent une large gamme de caractéristiques géographiques, morphologiques, géologiques et climatiques rencontrées dans la région alpine (Tab. 1). La fracture principale séparant les compartiments stable et instable est notée $F A$ (Fig. 2) et son orientation est indiquée dans le Tableau 2. Pour chaque site, l'instrumentation consiste à installer un (ou plusieurs) vélocimètres(s) courte période(s) à trois composantes (3-C) sur le compartiment instable accompagné(s) d'un capteur de référence sur le massif stable adjacent. Ceux-ci enregistrent le mouvement du sol en continu selon les axes Vertical, Nord-Sud et Est-Ouest. Les paramètres météorologiques (température, pluviométrie, etc.) ont été mesurés par une station installée sur site. Le temps universel coordonné (UTC) a été utilisé pour la synchronisation des données. La durée d'acquisition de données en continu (Tab. 2) varie d'environ 2 ans à 9 ans selon les sites et les contraintes (durée des projets, disponibilité du matériel, etc.). Les signaux de vibrations ambiantes de chaque capteur sont ensuite traités pour calculer la distribution azimutale de l'énergie du bruit de fond sismique pour chaque heure d'enregistrement. Ces résultats sont présentés sur la Figure 3. Deux diagrammes azimutaux sont présentés pour chaque site, utilisant la même échelle de couleurs : un pour le 
Tableau 1. Caractéristiques des sites d'étude.

Table 1. Study sites characteristics.

\begin{tabular}{llllll}
\hline Site & Climat & Géologie & Volume $\left(\mathrm{m}^{3}\right)$ & Morphologie & Méca. rupture \\
\hline Rubi & Méditerranéen dégradé & Pélites & 4500 & Colonne & B/G \\
La Suche & Semi-continental & Calcaires massifs & 30000 & Colonne & B/G \\
La Praz & Montagnard & Grès et schistes & 13000 & Versant fracturé & Glissement composé \\
Les Arches & Montagnard & Calcaires biolastiques ${ }^{\mathrm{c},}$, & 1000 & Colonne & B/G \\
\hline
\end{tabular}

$\mathrm{B} / \mathrm{G}$ : basculement ou glissement en pied.

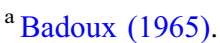

${ }^{\mathrm{b}}$ Debelmas et al. (1989).

c Arnaud et al. (1974).

${ }^{\mathrm{d}}$ Lévy et al. (2010).

Tableau 2. Caractéristiques des pics spectraux $f_{0}$ pour chaque site.

Table 2. Site properties and recorded spectral peaks $f_{0}$ characteristics.

\begin{tabular}{|c|c|c|c|c|c|}
\hline Site & Volume & $f_{0}(\mathrm{~Hz})$ & Azimut $F A\left({ }^{\circ} / \mathrm{N}\right)$ & Azimut $f_{0}(\% / \mathrm{N})$ & Acquisition \\
\hline Rubi & 4500 & 5 & 65 & 160 & 2011-2013 \\
\hline La Suche & 30000 & 2,3 & Fracturation multiple & 15 & 2011-2013 \\
\hline Les Arches & 1000 & 6,3 & 145 & 54 & 2008-en cours \\
\hline
\end{tabular}

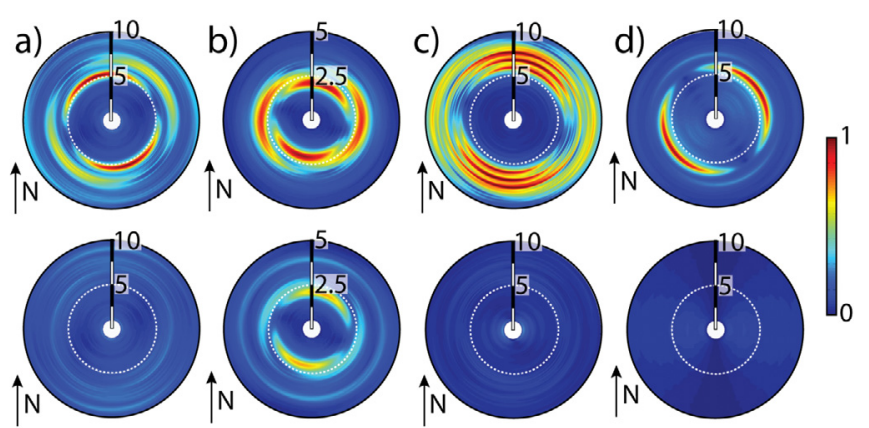

Fig. 3. Distribution azimutale de l'énergie des vibrations ambiantes sur les 4 sites. (Haut) Enregistrements sur le compartiment instable. (Bas) Mesures sur le massif stable. La distance radiale correspond à l'axe des fréquences. Le Nord est orienté vers le haut du diagramme (flèche noire). L'échelle de couleur indique l'amplitude de la transformée de Fourier, normalisée à 1 pour chaque site : $a$ : Rubi ; $b$ : La Suche; c: La Praz; d: Les Arches.

Fig. 3. Azimutal distribution of ambient vibration spectra at the 4 potential rockfall sites. (Top) Seismic records on the unstable rock mass and (Bottom) records on nearby stable rock mass. The circle radius shows the frequency axis. North direction is oriented upwards. The colorscale shows the Fourier transform amplitude normalized to one for each site: $a$ : Rubi; b: La Suche; c: La Praz; d: Les Arches.

compartiment instable (ligne supérieure) et un pour le massif stable (ligne inférieure). Les diagrammes sont calculés sur une semaine d'enregistrement de vibrations ambiantes.

La Figure 3 montre que des pics spectraux sont observés systématiquement sur les compartiments instables (haut) contrairement au massif stable adjacent (bas), dans des directions de vibration bien spécifiques et à certaines fréquences. Pour le site de La Suche (Fig. 3b), le capteur de référence est situé à proximité immédiate du compartiment instable et enregistre probablement une partie de sa vibration. Cela explique la présence des mêmes pics spectraux sur les deux capteurs. La fréquence du pic spectral principal montre globalement une corrélation négative avec le volume de l'instabilité. Ces pics sont interprétés comme les fréquences de résonance du compartiment instable, en accords avec les modélisations numériques réalisées (Bottelin et al., 2013). Le pic spectral principal correspond à la fréquence de résonance fondamentale du compartiment instable $f_{0}$. On observe également une direction de vibration préférentielle à la fréquence $f_{0}$, dont l'azimut est indiqué dans le Tableau 2. Pour les sites pour lesquels il existe une fracture arrière bien définie (Rubi, La Praz, Les Arches), la direction de vibration à $f_{0}$ est systématiquement perpendiculaire à la fracture arrière, selon la ligne de plus grande pente.

\subsection{Variations temporelles d'origine thermique}

Dans un second temps, nous avons calculé la Densité Spectrale de Puissance au cours du temps, afin de suivre la fréquence de résonance fondamentale $f_{0}$. L'ensemble des compartiments rocheux instables étudiés ont montré des fluctuations réversibles de la fréquence fondamentale d'amplitude très variables. Ces fluctuations réversibles sont gênantes, car elles pourraient masquer une chute irréversible de $f_{0}$ liée à de l'endommagement. Le suivi de $f_{0}$ est représenté sur la Figure $4 \mathrm{a}$ et $\mathrm{b}$ pour les sites des Arches et La Praz, respectivement. Ces sites sont présentés car ils montrent respectivement les amplitudes de variations de $f_{0}$ les plus faibles et les plus importantes. Pour chaque site, les données de température et de précipitation sont présentées en regard du suivi de $f_{0}$. On observe des variations de $f_{0}$ selon plusieurs échelles temporelles: journalière et pluri-journalière. Ces 
a)

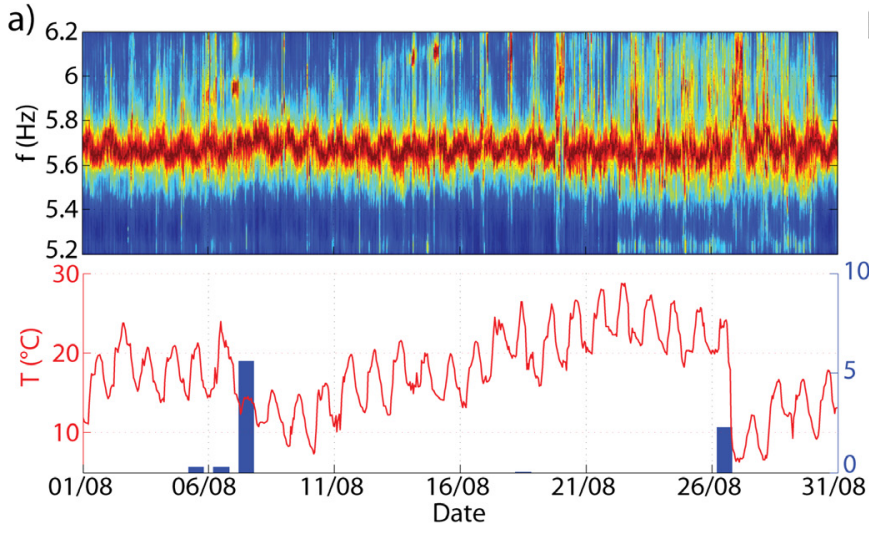

b)

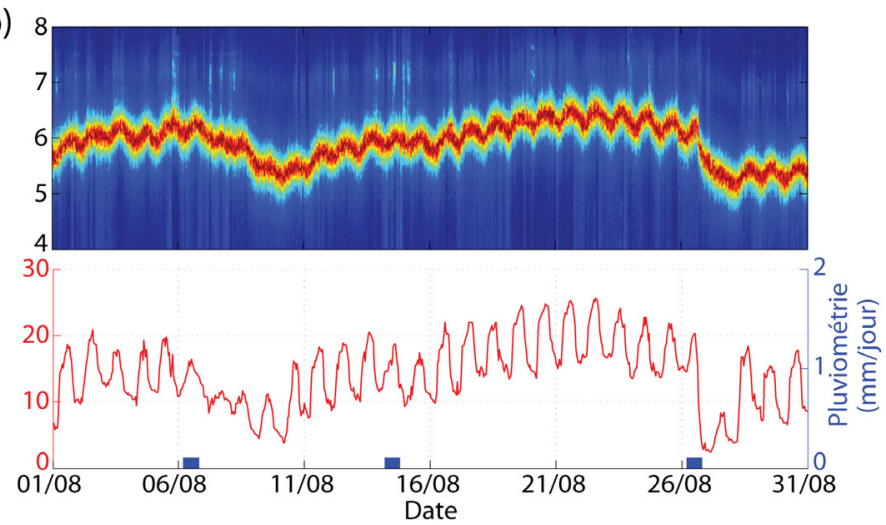

Fig. 4. (Haut) Suivi temporel des densités spectrales de puissance normalisées du bruit de fond enregistré sur le compartiment instable, pour les sites de La Praz (a) et des Arches (b), en août 2011. La direction représentée est la direction de vibration à $f_{0}$ (Tab. 2). (Bas) Température de l'air (rouge) et pluviométrie (bleu) mesurées sur les sites.

Fig. 4. (Top) Ambient vibration spectral monitoring over August 2011. Normalized Power Spectral Density of seismic records on the unstable rock mass for La Praz (a) and Les Arches (b) sites. The direction of monitoring corresponds to the direction of vibration at $f_{0}$ (see Tab. 2). (Bottom) Air temperature (red) and rainfall (blue) at La Praz (a) and Les Arches (b), over the same period.

fluctuations de $f_{0}$ apparaissent très fortement corrélées avec les variations de température (Fig. 4a et b). Cette corrélation peut être importante et de signe positif (Les Arches, Fig. 4b) ou moins marquée et de signe négatif (La Praz, Fig. 4a). Le lien entre la température et $f_{0}$ semble donc complexe, dépendant du site et de l'échelle de temps étudiée. Le site des Arches (Fig. 4a) a été investigué plus en profondeur en raison de la longue série temporelle de données disponibles (plus de deux ans). De plus, sa fréquence fondamentale est la plus sensible aux effets thermiques. Nous avons pu expliquer les variations multi-temporelles de $f_{0}$ par la conjugaison de plusieurs effets thermiques :

- le module d'élasticité de la roche varie de quelques pour cents en fonction de la température, et la profondeur affectée par les changements thermiques dépend de la période de sollicitation (journalier, pluri-journalier, saisonnier);

- la contraction/dilatation du matériau permet la modification de la raideur du contact au niveau des ponts rocheux;

- la formation de glace dans la fracture arrière $F A$ qui sépare la masse instable du massif rocheux augmente temporairement la cohésion au niveau de la future surface de rupture.

L'étude détaillée de ces phénomènes thermo-mécaniques est disponible dans Bottelin et al. (2013). Ces mécanismes ont pu être transposés à l'ensemble des sites d'étude et permettent d'expliquer les fluctuations réversibles de $f_{0}$. Durant la période d'instrumentation des sites (Tab. 2), aucun endommagement irréversible n'a pu être mesuré.

\section{Glissements argileux}

\subsection{Expérience analogique}

Nous avons cherché à tester la mesure de $V_{S}$ comme indicateur de l'état structurel des argiles sur un modèle analogique de glissement de terrain argileux. Pour cela, nous avons construit un plan incliné pouvant contenir un volume décimétrique d'argile. Les argiles utilisées ont été saturées en eau, i.e. avec une teneur en eau gravimétrique (rapport entre la masse d'eau et celle de la fraction solide) au-dessus de la limite de liquidité $w_{L}$ déterminée à environ 0,44 (Mainsant et al., 2012a). Le glissement en surface de l'argile a été mesuré en trois points $(D 1, D 2, D 3$, Fig. 5). Nous avons mesuré en continu lors de l'expérience la vitesse des ondes de Rayleigh $V_{R}$ à l'aide d'une source piézoélectrique et de capteurs accélérométriques. La mesure a été réalisée dans la bande de fréquence $(100-600 \mathrm{~Hz})$, qui permet de prospecter l'épaisseur entière de la masse argileuse. Le plan a été incliné progressivement de $1^{\circ}$ par minute. Les résultats de deux expériences réalisées à deux teneurs en eau différentes et sont présentés sur la Figure $5 \mathrm{a}$ et $\mathrm{b}$, respectivement.

La première expérience $(w=0,57)$ montre une $V_{R}$ initiale de $12,1 \mathrm{~m} / \mathrm{s}$ jusqu'à ce que le plan atteigne un angle $\theta$ de $8^{\circ}$ au temps $t_{l}$ (Fig. 5a). Ensuite, $V_{R}$ diminue progressivement alors qu'aucun mouvement n'est détecté par les marqueurs de surface. Cette diminution relative atteint $10 \%$ juste avant $t_{2}$. À $t_{2}$, le glissement argileux accélère nettement. Lors de cette phase, la déstructuration du matériau devient très importante et la vitesse des ondes de surface n'est plus interprétable (non illustré). À $t_{3}$, le plan incliné commence à redescendre et le mouvement s'arrête.

La seconde expérience $(w=0,68)$ montre une $V_{R}$ initiale plus faible que lors de l'expérience précédente $(8,7 \mathrm{~m} / \mathrm{s}) . V_{R}$ commence à diminuer pour un angle plus faible de $\theta=5^{\circ}$ à $t_{I}$ (Fig. 5b). À nouveau, aucun mouvement de la masse argileuse n'est détecté à ce stade. $V_{R}$ connaît une baisse totale d'environ $7 \%$ pour atteindre $8,1 \mathrm{~m} / \mathrm{s}$. À $t_{2}$ pour un angle de $\theta=9^{\circ}$, la surface commence à se déplacer à une vitesse constante. Les ondes de surface ne sont alors plus reconstructibles et $V_{R}$ n'est donc plus interprétable. À $t_{2}$,' le pied du glissement touche le fond du plan incliné et les mesures de mouvement de la masse argileuse sont biaisées. À $t_{3}$, le plan incliné commence à redescendre et tout mouvement s'arrête. Ces deux expériences montrent systématiquement une chute significative de $V_{R}$ $(10 \%$ et $7 \%$, respectivement) avant tout déplacement significatif du matériau. Ces expériences de plan incliné confirment la faisabilité de la mesure de $V_{S}$ sur un échantillon d'argile décimétrique, avec des valeurs de $V_{S}$ très faibles pour des échantillons au-delà de la limite de liquidité $w_{L}$. Nos résultats attestent le caractère précurseur de la chute de $V S_{R}$ 

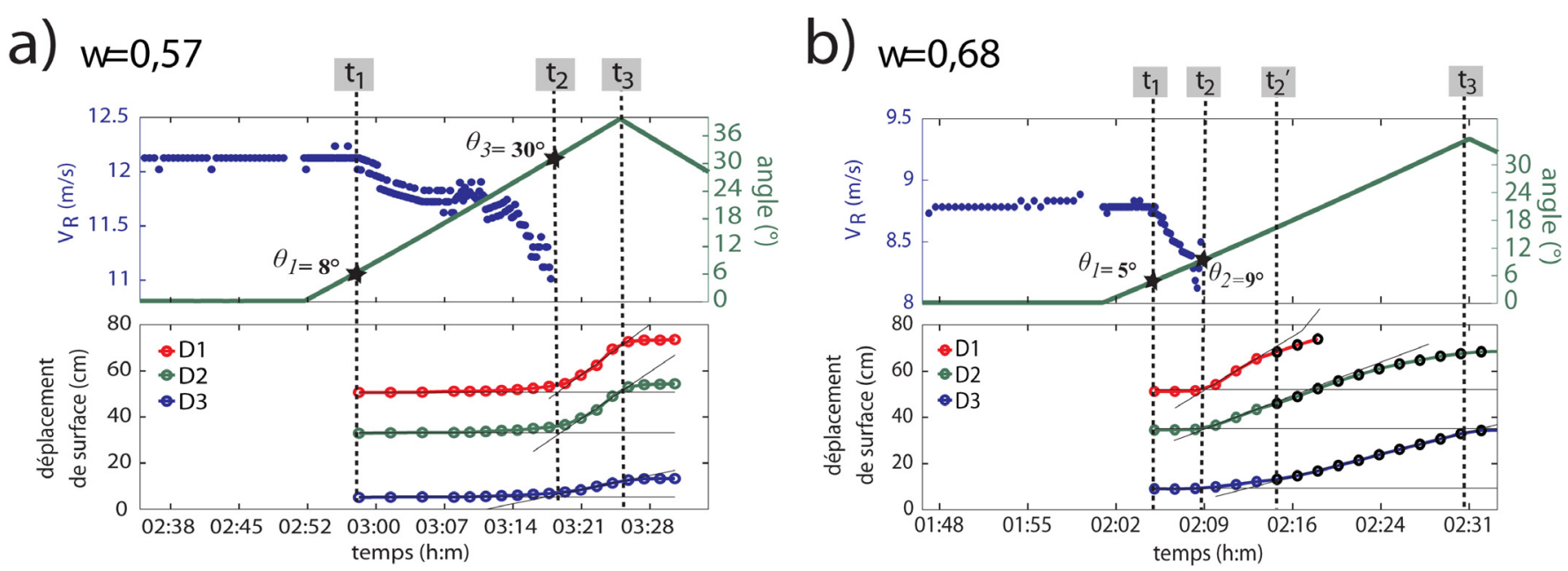

Fig. 5. Expériences de plan incliné : a : teneur en eau $w=0,57 ; \mathrm{b}:$ teneur en eau $w=0,68$. (Haut) Vitesse des ondes de Rayleigh $\left(V_{R}, \operatorname{points}\right.$ bleus) et angle du plan incliné (ligne verte) en fonction du temps. (Bas) Déplacements de surface mesurés aux points $D 1, D 2$ et $D 3$. $t_{1}, t_{2}$, $t_{2}$ ' et $t_{3}$ repèrent des temps caractéristiques ( $c f$. texte).

Fig. 5. Laboratory inclined plane experiments: $a$ : water content $w=0.57 ; b: w=0.68$. (Top) Rayleigh wave velocity ( $V_{R}$, blue dots) and plane inclination (green line) over time. (Bottom) Surface displacement at D1, D2 and D3 markers. $t_{1}, t_{2}, t_{2}$ ' and $t_{3}$ points show the characteristic times described in the main text.

(ou $V_{R}$, indifféremment) au glissement. On note que plus la teneur en eau des argiles est faible, plus la phase transitoire de la réponse de $V_{S}$ est longue.

\subsection{Surveillance du site expérimental}

L'instrumentation et la surveillance du glissement argileux du Pont-Bourquin (Suisse) ont été réalisées au début de l'année 2010. Ce glissement de terrain long de $100 \mathrm{~m}$, large d'environ $40 \mathrm{~m}$ et profond de $11 \mathrm{~m}$ menace la route reliant les Diablerets à Gstaad (Jaboyedoff et al., 2009) (Fig. 6a). Il évolue par épisodes de glissements-coulées successifs, dont un épisode significatif s'est produit le 18 août 2010 et a mobilisé entre 3000 et $6000 \mathrm{~m}^{3}$ de matériau. Deux capteurs vélocimétriques verticaux une composante ont été placés de part et d'autre du glissement dans des zones stables (Fig. 6a). Ils se trouvent au niveau du bourrelet principal du glissement, la où la contrainte verticale exercée par l'apport successif de terrain augmente au cours du temps (Mainsant et al., 2012b). Les vibrations ambiantes enregistrées se sont révélées suffisamment stables dans l'espace et dans le temps pour négliger des effets de source. Les variations de vitesse des ondes de surface sont donc attribuables uniquement à un changement d'état du milieu de propagation. Les ondes de surface se propageant entre les deux capteurs ont été reconstruites par corrélation des vibrations ambiantes enregistrées heure par heure (Gouedard et al., 2008). Ces corrélations horaires ont ensuite été moyennées sur une journée. La corrélation dite de référence a été arbitrairement fixée comme la moyenne des corrélations journalières sur la période de surveillance. Les variations relatives de vitesse des ondes de surface (notées $d V / V$ ) sont obtenues en comparant le décalage temporel entre la corrélation journalière et la corrélation de référence. En complément de l'instrumentation sismique, un piézomètre a été installé au centre du glissement ainsi qu'une station météo en partie haute (Fig. 6a).

La surveillance sismique passive est présentée sur la période allant du $1^{\mathrm{er}}$ avril au 23 août 2010 (Fig. 6b). La variation relative de vitesse des ondes de surface $(d V / V)$ dans la bande $10-12 \mathrm{~Hz}$ est indiquée en bleu. On observe une légère augmentation de vitesse d'environ $2 \%$ durant les 4 premiers mois de la surveillance. Du $1^{\mathrm{er}}$ avril jusqu'à début mai, le niveau piézométrique dans le corps du glissement est relativement bas (courbe verte, Fig. 6b). Ce niveau augmente rapidement durant le mois de mai, en raison de la fonte des neiges et de précipitations régulières (courbe rouge, Fig. 6b). À partir des fortes précipitations du 24 juillet, la vitesse des ondes de surface diminue progressivement d'environ $2 \%$ jusqu'au 15 août. Après le 15 août, $d V / V$ montre une nouvelle chute d'environ $5 \%$ jusqu'à la coulée du 18 août 2010. Une diminution totale de $V_{S}$ d'environ $7 \%$ a pu ainsi être détectée au sein du glissement, plusieurs semaines avant le mouvement de terrain.

Afin de mieux connaître les propriétés sismiques du corps du glissement et interpréter la chute de $V_{S}$, une campagne de sismique active a été réalisée entre les deux capteurs (Fig. 6a). Le profil de vitesse des ondes de cisaillement $V_{S}$ du glissement a ainsi été établi (Fig. 6c). En surface, $V_{S}$ est faible $(\approx 90 \mathrm{~m} / \mathrm{s})$ sur les deux premiers mètres et présente de fortes variations latérales en raison des nombreuses fissures présentes (Mainsant et al., 2012b). Cette valeur est en bon accord avec les matériaux argileux très meuble, fracturé et fortement hydraté observé en surface sur le terrain. Ensuite, $V_{S}$ atteint $360 \mathrm{~m} / \mathrm{s}$ dans le corps du glissement. Le substratum a été détecté à environ $11 \mathrm{~m}$ de profondeur, dans lequel $V_{S}$ atteint $660 \mathrm{~m} / \mathrm{s}$. Nous avons ajouté à ce modèle de sol une zone de déformation basale, pour laquelle nous avons fait varier son épaisseur et la vitesse des ondes de cisaillement ( $H$ et $V_{S 2 \text { bis }}$, Fig. $6 \mathrm{c}$ ). Nous présentons les cinq courbes de variations relatives de vitesses des ondes de surface qui approchent le mieux les mesures de sismique passive (Fig. 6c). Le plus faible écart entre les mesure et le modèle est atteint pour une couche basale de $\sim 2,5 \mathrm{~m}$ d'épaisseur, dont la vitesse $V_{S 2 \text { bis }}$ diminue de $360 \mathrm{~m} / \mathrm{s}$ à $200 \mathrm{~m} / \mathrm{s}$ entre le début de la surveillance et le glissement-coulée. Le noyau de 

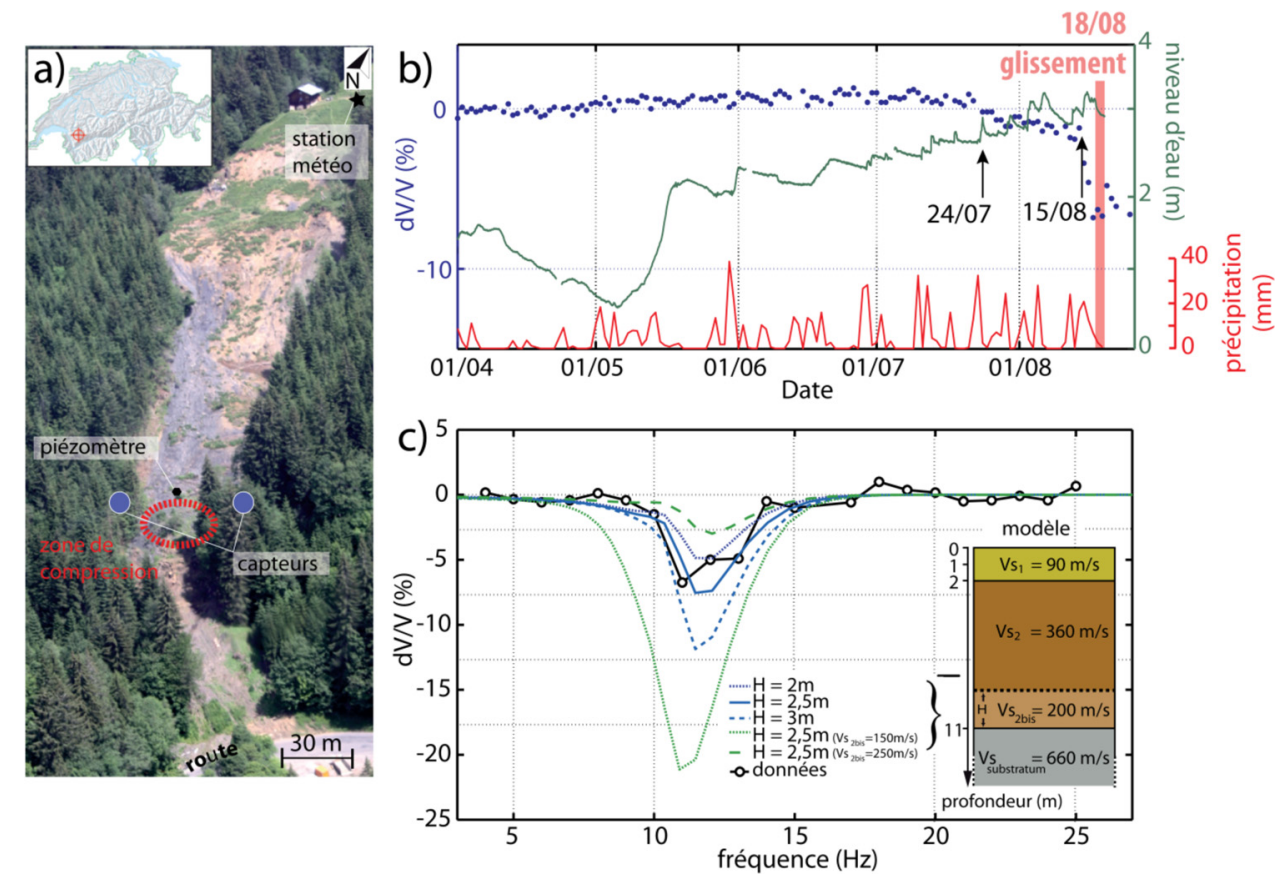

Fig. 6. Instrumentation du glissement du Pont-Bourquin (Suisse) et résultats de la surveillance sismique : a : vue aérienne du glissement argileux et localisation des instruments ; $\mathrm{b}$ : variation relative des vitesses des ondes de surface ( $d V / V$, points bleus), du niveau d'eau (courbe verte) et des précipitations (courbe rouge) durant l'année $2010 ; \mathrm{c}: d V / V$ en fonction de la fréquence. Courbe noire: observations de $d V / V$ sur le site expérimental. Courbes bleues: étude de l'influence de l'épaisseur $H$ de la couche basale, avec $V_{S 2 b i s}=200 \mathrm{~m} / \mathrm{s}$. Courbes vertes: étude de l'influence de la vitesse $V_{S 2 b i s}$ de la couche basale, pour une épaisseur de 2,5 m. Le modèle présentant la plus faible erreur est indiqué par la courbe bleue continue. Le modèle de sol (coupe) est schématisé en bas à droite.

Fig. 6. Pont Bourquin clayey landslide (Switzerland): instrumentation and seismic monitoring results: a: aerial view of the landslide. Seismic sensors are shown with blue dots. The clay accumulation zone is circled with red dashes; $b$ : relative surface wave velocity variations (dV/V, blue dots), piezometric level (green curve) and rainfall (red curve) over year 2010; $c$ : dV/V as a function of frequency. (Black) dV/Vobservations at Pont Bourquin landslide. $d V / V$ parametric study: influence of basal layer thickness $H$, with $V_{S 2 b i s}=200 \mathrm{~m} / \mathrm{s}$ (dashed blue) and influence of $V_{S 2 b i s}$ with $H=2.5 \mathrm{~m}$ (dashed green), respectively. The simulation best fitting the measurements is shown by the continuous blue curve. Corresponding layered soil section is shown at the bottom right corner.

sensibilité des ondes de surface a été calculé sur ce modèle de sol. Une diminution de $V_{S}$ dans la couche basale provoque une chute de la vitesse des ondes de surface autour de $11 \mathrm{~Hz}$, ce qui est cohérent avec le comportement observé de $d V / V$ dans la bande $10-12 \mathrm{~Hz}$.

À partir du comportement rhéologique du matériau observé en laboratoire et lors de l'expérience analogique du plan incliné, nous interprétons cette diminution de $d V / V$ dans le zone basale du glissement comme un marqueur de la phase transitoire de la bifurcation de viscosité des argiles. Durant la période $\mathrm{du} 1^{\mathrm{er}}$ avril jusqu'à la fin juillet 2010, la zone d'accumulation a connu un chargement progressif par des matériaux provenant de l'amont (Fig. 6a). Le niveau d'eau dans le corps du glissement augmente en parallèle, de mai à juillet (Fig. 6b). Les contraintes de cisaillement dans la couche basale du glissement s'accroissent progressivement à cause du poids des terres tandis que la contrainte seuil de bifurcation de viscosité des argiles diminue par l'augmentation de la teneur en eau. À partir de la fin juillet, la perte de rigidité durant la déstructuration des argiles de la couche basale du glissement se traduit par une baisse de la vitesse des ondes de cisaillement d'environ $7 \%$. Dans ce cas d'étude sur site réel, la chute de $V_{S}$ est un précurseur de plusieurs semaines par rapport au déclenchement du glissement-coulée.

\section{Conclusions}

Cet article présente les résultats de nos travaux de recherche sur quatre sites naturels d'éboulements rocheux et un glissement de terrain argileux. Ces instabilités sont caractérisées par une évolution temporelle brutale présentant peu de signes précurseurs. Nous avons instrumenté ces sites de manière à y enregistrer les vibrations sismiques ambiantes durant plusieurs années.

Pour les éboulements rocheux, l'analyse des vibrations ambiantes a systématiquement permis de déterminer la fréquence de résonance fondamentale du compartiment instable (notée $f_{0}$ ). Cette mesure de $f_{0}$ a été réalisée en continu, afin de détecter une éventuelle diminution irréversible de $f_{0}$, précurseur à l'éboulement. Cependant, nous avons mesuré des fluctuations réversibles de $f_{0}$ pouvant potentiellement masquer l'endommagement. Ces fluctuations sont principalement contrôlées par la température et dépendent de la période étudiée (de journalière à saisonnière). Des simulations numériques ont montré que la contraction-dilatation du matériau et la dépendance de son module élastique à la température permettent d'expliquer ces observations.

Pour les glissements de terrain argileux, les expériences analogiques sur plan incliné ont permis de valider le principe 
de la surveillance sismique. Une chute de la vitesse des ondes de cisaillement $V_{S}$ comprise entre 7 et $9 \%$ a été mesurée avant la mise en mouvement de la masse argileuse. Cette chute de vitesse a été interprétée comme marqueur de la déstructuration des argiles. Sur site réel, nous avons suivi l'évolution du glissement de Pont-Bourquin par vibrations ambiantes. Cette étude a montré une chute de $V_{S}$ d'environ $7 \%$, apparaissant avant les mouvements de surface importants. Cette chute de vitesse s'initie plusieurs semaines avant la coulée et s'accélère plusieurs jours avant l'évènement. Elle montre un caractère irréversible et précurseur. À partir du profil de vitesse du glissement, nous avons pu localiser la diminution de vitesse dans une zone basale de 2,5 $\mathrm{m}$ d'épaisseur. Nous interprétons cette modification de $V_{S}$ comme marqueur de la déstructuration de la couche d'argile basale du glissement, conséquence d'une augmentation de la teneur en eau du matériau et de l'accroissement de la contrainte cisaillante appliquée.

Ces résultats montrent le potentiel des vibrations ambiantes pour la surveillance de mouvements de terrain à déclenchement rapide. Pour les éboulements rocheux dans des roches rigides et pour le glissement de terrain argileux que nous avons instrumentés, le suivi sismique a apporté des informations globales, en profondeur et intégrées sur l'état mécanique de l'instabilité. Les efforts actuels que nous poursuivons visent à améliorer l'opérabilité de ces techniques en allégeant l'instrumentation nécessaire, en automatisant les processus de traitement et en développant des solutions pour s'affranchir des fluctuations de mesures d'origine thermique. Nos recherches ouvrent la voie vers l'utilisation de ces précurseurs sismiques comme complément aux méthodes de suivi classiques.

Remerciements. Ces travaux n'auraient pas vu le jour sans de nombreuses collaborations au sein d'ISTERRE et d'IRSTEA, Grenoble. Ils ont été principalement financés par l'ANR SISCA, le pôle VOR, les projets européens Mountain Risks et MASSA, le département de l'Isère à travers le Pôle grenoblois des risques naturels. Les auteurs remercient toutes les personnes ayant participé à l'effort d'instrumentation sur le terrain, les parcs régionaux et les laboratoires collaborateurs dont l'université de Lausanne et le laboratoire des transferts en hydrologie et environnement. Nous remercions les deux rapporteurs anonymes pour leur relecture attentive et leurs suggestions qui ont permis d'améliorer ce manuscrit.

\section{Conflits d'intérêts}

Les auteurs déclarent n'avoir aucun lien d'intérêt concernant les données publiées dans cet article.

\section{Références}

Abellán A, Jaboyedoff M, Oppikofer T, Vilaplana JM. 2009. Detection of millimetric deformation using a terrestrial laser scanner: experiment and application to a rockfall event. Nat Hazards Earth Syst Sci 9: 365-372. DOI: 10.5194/nhess-9-365-2009.

Abellán A, Calvet J, Vilaplana JM, Blanchard J. 2010. Detection and spatial prediction of rockfalls by means of terrestrial laser scanner monitoring. Geomorphology 119: 162-171.

Agliardi F, Crosta G. 2003. High resolution three-dimensional numerical modelling of rockfalls. Int J Rock Mech Min Sci 40: 455-471.

Amitrano D, Grasso JR, Senfaute G. 2005. Seismic precursory patterns before a cliff collapse and critical point phenomena. Geophys Res Lett 32: L08314.
Amitrano D, Arattano M, Chiarle M, et al. 2010. Microseismic activity analysis for the study of the rupture mechanisms in unstable rock masses. Nat Hazards Earth Syst Sci 10: 831-841.

Arnaud H, Meloux M, Montjuvent G. 1974. Notice explicative de la carte géologique de la France au 1/50 000ème, feuille XXXII-37 Mens (844). Bureau de Recherches Géologiques et Minières.

Azimi, C., \& Desvarreux, P. (1996). Quelques aspects de la prévision des mouvements de terrain. Revue française de géotechnique 76.

Badoux H. 1965. Notice explicative de la carte géologique de la France au 1/50 000ème, feuille XXXV-28 Thonon-Châtel. Bureau de Recherches Géologiques et Minières.

Bonnefoy-Claudet S, Cotton F, Bard P-Y. 2006. The nature of noise wavefield and its applications for site effects studies: A literature review. Earth Sci Rev 79:205-227. DOI: 10.1016/j.earsci rev.2006.07.004.

Bottelin P, Levy C, Baillet L, et al. 2013. Modal and thermal analysis of Les Arches unstable rock column (Vercors massif, French Alps). Geophys J Int 194: 849-858. DOI: 10.1093/gji/ggt046.

Bozzano F, Mazzanti P, Prestininzi A, Scarascia Mugnozza G. 2010. Research and development of advanced technologies for landslide hazard analysis in Italy. Landslides 7: 381-385. DOI: 10.1007/ s10346-010-0208-x.

Brenguier F, Campillo M, Hadziioannou C, et al. 2008a. Postseismic relaxation along the San Andreas fault at Parkfield from continuous seismological observations. Science 321: 1478-1481.

Brenguier F, Shapiro NM, Campillo M, et al. 2008b. Towards forecasting volcanic eruptions using seismic noise. Nat Geosci 1: 126-130.

Brückl E, Mertl S. 2006. Seismic monitoring of deep-seated mass movements. In: Proceedings of INTERPRAEVENT International Symposium Disaster Mitigation of Debris Flows, Slope Failures and Landslides, pp. 571-580.

Burjánek J, Gassner-Stamm G, Poggi V, et al. 2010. Ambient vibration analysis of an unstable mountain slope. Geophys J Int 180: 820-828.

Burjánek J, Moore JR, Molina Y, et al. 2012. Instrumental evidence of normal mode rock slope vibration. Geophys J Int 188: 559-569.

Casagrande A. 1932. Research on the Atterberg limits of soils. Public Roads 13: 121-136.

CFGI. 2000. Caractérisation et cartographie de l'aléa dû aux mouvements de terrain. Laboratoire Central des Ponts et Chaussées. 91 pages. Coll. Environnement : les risques naturels. Paris : Impr. REGICAR.

Cornforth DH. 2005. Landslides in practice. Wiley Hoboken.

Coussot P, Nguyen QD, Huynh HT, Bonn D. 2002. Viscosity bifurcation in thixotropic, yielding fluids. J Rheol 46: 573-589.

Crosta G, Agliardi F. 2003. Failure forecast for large rock slides by surface displacement measurements. Can Geotech J 40: 176-191.

Crozier MJ. 1999. Prediction of rainfall-triggered landslides: A test of the antecedent water status model. Earth Surf Process Landf 24: $825-833$.

Debelmas J, Desmons J, Ellenberger F, et al. 1989. Notice explicative de la carte géologique de la France au 1/50 000ème, feuille Modane (775). Bureau de Recherches Géologiques et Minières.

Duputel Z, Ferrazzini V, Brenguier F, et al. 2009. Real time monitoring of relative velocity changes using ambient seismic noise at the Piton de la Fournaise volcano (La Réunion) from January 2006 to June 2007. J Volcanol Geotherm Res 184: 164-173.

Frayssines M. 2005. Contribution à l'évaluation de l'aléa éboulement rocheux (rupture). Université Joseph-Fourier-Grenoble I.

Frayssines M, Hantz D. 2006. Failure mechanisms and triggering factors in calcareous cliffs of the Subalpine Ranges (French Alps). Eng Geol 86: 256-270.

Fukuzono T. 1985. A new method for predicting the failure time of a slope. In: Proceedings of the 4th International Conference and Field Workshop in Landslides, Tokyo, pp. 145-150. 
Gaffet S, Guglielmi Y, Cappa F, et al. 2010. Use of the simultaneous seismic, GPS and meteorological monitoring for the characterization of a large unstable mountain slope in the southern French Alps. Geophys J Int 182: 1395-1410.

Gouedard P, Stehly L, Brenguier F, et al. 2008. Cross-correlation of random fields: Mathematical approach and applications. Geophys Prospect 56: 375-393.

Gunzburger Y, Merrien-Soukatchoff V, Guglielmi Y. 2005. Influence of daily surface temperature fluctuations on rock slope stability: case study of the Rochers de Valabres slope (France). Int $J$ Rock Mech Min Sci 42: 331-349.

Gutenberg B. 1958. Microseisms, Advances in Geophysics, Vol. 5, pp. 53-92, ISSN 0065-2687.

Helmstetter A, Garambois S. 2010. Seismic monitoring of Séchilienne rockslide (French Alps): Analysis of seismic signals and their correlation with rainfalls. Journal of Geophysical Research: Earth Surface 115 (F3).

Hovius N, Meunier P. 2012. Landslides: Earthquake ground motion and patterns of seismically induced landsliding. In: Clague J.J, Stead D, eds. Landslides: types, mechanisms and modeling. Cambridge, Great Britain: Cambridge University Press, pp. 24-36.

Huang X, Garcia MH. 1998. A Herschel-Bulkley model for mud flow down a slope. J Fluid Mech 374: 305-333.

Iverson RM. 2000. Landslide triggering by rain infiltration. Water Resour Res 36:1897-1910. DOI: 10.1029/2000WR900090.

Jaboyedoff M, Pedrazzini A, Loye A, et al. 2009. Earth flow in a complex geological environment: the example of Pont Bourquin, Les Diablerets (Western Switzerland). In: Proceedings Landslide Process Geo morphol Mapp Dyn Model, Chapter 2: Landslide mechanics \& mechanisms with focus on hydrological processes, pp. 131-137.

Jongmans D, Bievre G, Renalier F, et al. 2009. Geophysical investigation of a large landslide in glaciolacustrine clays in the Trièves area (French Alps). Eng Geol 109: 45-56.

Khaldoun A, Moller P, Fall A, et al. 2009. Quick clay and landslides of clayey soils. Phys Rev Lett 103: 188301.

Lacroix P, Helmstetter A. 2011. Location of seismic signals associated with microearthquakes and rockfalls on the Séchilienne landslide, French Alps. Bull Seismol Soc Am 101: 341-353.

Lévy C, Baillet L, Jongmans D, et al. 2010. Dynamic response of the Chamousset rock column (Western Alps, France). J Geophys Res DOI: $10.1029 / 2009 J F 001606$.

Lévy C, Jongmans D, Baillet L. 2011. Analysis of seismic signals recorded on a prone-to-fall rock column (Vercors massif, French Alps). Geophys J Int 186: 296-310.

Mainsant G, Jongmans D, Chambon G, et al. 2012a. Shear-wave velocity as an indicator for rheological changes in clay materials: Lessons from laboratory experiments. Geophys Res Lett 39: L19301. DOI: 10.1029/2012GL053159.

Mainsant G, Larose E, Brönnimann C, Jongmans, D, Michoud C, Jaboyedoff M, 2012b. Ambient seismic noise monitoring of a clay landslide: Toward failure prediction. Journal of Geophysical Research: Earth Surface 117(F1).

Matsuoka N. 2001. Direct observation of frost wedging in alpine bedrock. Earth Surf Process Landf 26: 601-614.

Moore JR, Gischig V, Burjanek J, et al. 2011. Site effects in unstable rock slopes: dynamic behavior of the Randa instability (Switzerland). Bull Seismol Soc Am 101: 3110-3116.

Mucciarelli M, Gallipoli MR, Di Giacomo D, et al. 2005. The influence of wind on measurements of seismic noise. Geophys J Int 161: 303-308.
Mufundirwa A, Fujii Y, Kodama N, Kodama J. 2011. Analysis of natural rock slope deformations under temperature variation: A case from a cool temperate region in Japan. Cold Reg Sci Technol 65: 488-500.

Obermann A, Kraft T, Larose E, Wiemer S. 2015. Potential of ambient seismic noise techniques to monitor the St. Gallen geothermal site (Switzerland). J Geophys Res Solid Earth 120: 4301-4316.

Occhiena C, Coviello V, Arattano M, et al. 2012. Analysis of microseismic signals and temperature recordings for rock slope stability investigations in high mountain areas. Nat Hazards Earth Syst Sci 12: 2283-2298.

Oppikofer T, Jaboyedoff M, Blikra L, et al. 2009. Characterization and monitoring of the $\backslash$ AAknes rockslide using terrestrial laser scanning. Nat Hazards Earth Syst Sci 9: 1003-1019.

Petley DN. 2013. Characterizing Giant Landslides. Science 339: 1395-1396.

Renalier F, Bièvre G, Jongmans D, et al. 2010. Clayey landslide investigations using active and passive Vs measurements. Adv Surf Seismol Ground-Penetrating Radar Geophys Dev Ser 15: 397-414.

Rosser N, Lim M, Petley D, et al. 2007. Patterns of precursory rockfall prior to slope failure.

Saito M. 1980. Semi-logarithmic representation for forecasting slope failure. In: Proceedings of the International Symposium on Landslides New Dehli, Vol. 1, pp. 321-324.

Schuster RL. 1996. Landslide: investigation and mitigation. In Keith TA, Schuster RL, eds.Transportation Research Board Special Report. Transport Research Board Special Report 247, National Academy Press, pp. 12-35.

Senfaute G, Duperret A, Lawrence J. 2009. Micro-seismic precursory cracks prior to rock-fall on coastal chalk cliffs: a case study at Mesnil-Val, Normandie, France. Nat Hazards Earth Syst Sci 9: 1625-1641.

Sornette D, Helmstetter A, Andersen J, et al. 2004. Towards landslide predictions: two case studies. Phys Stat Mech Its Appl 338: 605-632.

Spillmann T, Maurer H, Green AG, et al. 2007. Microseismic investigation of an unstable mountain slope in the Swiss Alps. $J$ Geophys Res 112: B07301.

Suwa H, Mizuno T, Ishii T. 2010. Prediction of a landslide and analysis of slide motion with reference to the 2004 Ohto slide in Nara, Japan. Geomorphology 124: 157-163.

Trappe V, Prasad V, Cipelletti L, et al. 2001. Jamming phase diagram for attractive particles. Nature 411: 772-775.

Viktorov IA. 1970. Rayleigh and Lamb waves: physical theory and applications. New York: Plenum Press, Coll. Ultrasonic technology, $154 \mathrm{p}$.

Vilajosana I, Surinach E, Abellan A, et al. 2008. Rockfall induced seismic signals: case study in Montserrat, Catalonia. Nat Hazards Earth Syst Sci 8: 805-812.

Voight B. 1989. A Relation to Describe Rate-Dependent Material Failure. Science 243:200-203. DOI: 10.1126/science.243.4888.200.

Wilkinson PL, Anderson MG, Lloyd DM. 2002. An integrated hydrological model for rain-induced landslide prediction. Earth Surf Process Landf 27: 1285-1297.

Withers MM, Aster RC, Young CJ, Chael EP. 1996. High-frequency analysis of seismic background noise as a function of wind speed and shallow depth. Bull Seismol Soc Am 86: 1507-1515.

Zvelebill J, Moser M. 2001. Monitoring based time-prediction of rock falls: three case-histories. Phys Chem Earth Part B Hydrol Oceans Atmosphere 26: 159-167.

Citation de l'article : Pierre Bottelin, Guénolé Mainsant, Denis Jongmans, Laurent Baillet, Eric Larose, Guillaume Chambon, Didier Hantz. Suivi temporel de mouvements gravitaires : apport des vibrations sismiques ambiantes. Rev. Fr. Geotech. 2017, $152,1$. 